\title{
High levels of neurological involvement but low mortality in miliary tuberculosis: a 6-year case-series from the UK
}

\author{
To the Editor:
}

Tuberculosis (TB) remains one of the biggest global health challenges. Whilst the greatest burden of active disease is seen in Asia and Africa [1], TB remains a significant issue in the UK. Miliary TB is one of the severest manifestations of TB disease [2-4]. Up-to-date clinicopathological data on miliary TB from the developed world are lacking. We undertook a comprehensive 6-year review (2007-2012) of cases presenting to a single UK centre with an ethnically diverse population with high levels of population exchange with the Indian Subcontinent and Africa. Miliary TB was defined as the presence of miliary nodules on thoracic imaging in patients who presented with symptoms compatible with the diagnosis and either culture of Mycobacterium tuberculosis complex or culture-negative patients with clinical and/or histological features compatible with TB who were commenced on a course of antituberculous therapy (ATT).

42 cases were included in the final analysis (median age 48.5 years and 59.5\% males). Most miliary TB cases occurred in foreign-born patients from the Indian subcontinent; $60 \%$ of cases in migrants occurred within 5 years of arrival to the UK. HIV testing was performed in 38 (90.5\%) out of 42 patients and four (10.5\%) out of 38 patients were positive (all foreign-born: Sub-Saharan African, n=3; Indian Subcontinent, n=1).

Overall mortality was $7.1 \%$ (three out of 42 patients); two patients died during their course of ATT and one patient died before starting treatment. All patients had samples sent for microbiological culture with bronchoalveolar lavage (BAL) (27 (64.3\%) out of 42 patients) and sputum (25 (59.5\%) out of 42 patients) being the most commonly positive samples. The organism was isolated in $32(76.2 \%)$ out of 42 patients. BAL and sputum specimens were culture positive in 15 (55.6\%) out of 27 patients and 18 (72\%) out of 25 patients, respectively. Four (9.5\%) out of 42 patients were smear-positive for acid fast bacilli. All organisms were fully sensitive to first-line ATT. When we examined the monocyte:lymphocyte ratio we found that it was raised at presentation/pre-treatment (median 0.47 (interquartile range) $0.33-0.73$ ) and significantly decreased at the end of treatment $(0.25(0.16-0.27) ; \mathrm{p}=0.0001)$; this was largely driven by the resolution of lymphopenia.

Assessment of central nervous system (CNS) involvement with a lumbar puncture and/or neuroimaging was undertaken in a high proportion (39 (92.9\%) out of 42 ) of patients (table 1). Of these 39 patients, 28 (71.8\%) underwent neuroimaging and $31(79.5 \%)$ had a lumbar puncture performed (table 1$)$. We found a relatively high level $(38.5 \%)$ of neurological involvement, as defined by radiological or cerebrospinal fluid (CSF) evidence of CNS disease (table 1). In our analysis, magnetic resonance imaging (MRI) scans were significantly more likely to be abnormal than computed tomography (CT) scans (13 (65\%) out of 20 patients versus two $(14 \%)$ out of 14 patients $(\mathrm{p}=0.003)$ ), particularly in identifying tuberculomas which were seen in $55 \%$ of cases. Just under three-quarters (31 out of 42 ) of patients in our study underwent a lumbar puncture; this is a much higher proportion in comparison to previous studies. CSF abnormalities were only detected in five patients overall. Only one patient with normal neuroimaging had an abnormal CSF; patients with an abnormal CSF did, however, have a high yield for culture positivity.

Sensitivity for identifying CNS involvement for the different modalities (CT, MRI and lumbar puncture) is outlined in table 1. Overall, MRI was significantly more sensitive (92.9\%, 95\% CI 66.1-99.8\%) than both CT $(40.0 \%, 95 \%$ CI 5.3-85.3\%; $\mathrm{p}=0.013)$ and lumbar puncture $(41.7 \%, 95 \%$ CI $15.2-72.3 \%, \mathrm{p}=0.005)$. Amongst 12 patients with CNS involvement who were investigated with both neuroimaging and lumbar puncture, five (41.7\%) had symptoms/signs of neurological involvement. All five patients underwent a lumbar puncture and neuroimaging (as per National Institute for Health and Clinical Excellence (NICE) guidelines) [5]. There were three (60\%) abnormal lumbar punctures and four (80\%) patients had abnormal neuroimaging $(\mathrm{p}=1.0)$. In the seven asymptomatic patients, where NICE recommends lumbar puncture only [5], lumbar puncture was only abnormal in two (28.6\%) patients whereas neuroimaging was abnormal in all seven $(100 \%)$ patients $(\mathrm{p}=0.02)$.

This study does have a number of limitations. It is a relatively small, retrospective cohort of patients from a single regional centre in the UK. However, our region does have a relatively high fraction of national TB cases and the study does give insight into the investigation and outcome of patients with miliary TB in a developed world setting. We also found no evidence of temporal change in the clinical management of miliary TB patients. The overall mortality (7.1\%) was low in comparison to other modern case series and 
TABLE 1 Demographic characteristics of 42 individuals with miliary tuberculosis

\section{Variable}

\begin{tabular}{|c|c|}
\hline Age years & $48.5(29.0-65.0)$ \\
\hline \multicolumn{2}{|l|}{ Age categories years } \\
\hline $16-25$ & $7(16.7)$ \\
\hline $26-35$ & $7(16.7)$ \\
\hline $36-45$ & 5 (11.9) \\
\hline $46-55$ & $9(21.4)$ \\
\hline$>55$ & $14(33.3)$ \\
\hline \multicolumn{2}{|l|}{ Sex } \\
\hline Female & $17(40.5)$ \\
\hline Male & 25 (59.5) \\
\hline \multicolumn{2}{|l|}{ Ethnicity } \\
\hline White & $3(7.1)$ \\
\hline Indian Subcontinent & $34(80.9)$ \\
\hline Afro-Caribbean & $5(11.9)$ \\
\hline \multicolumn{2}{|l|}{ Place of birth } \\
\hline UK & $3(7.1)$ \\
\hline Foreign born & 39 (92.9) \\
\hline \multicolumn{2}{|l|}{ Time since entry to the UK years ${ }^{\#}$} \\
\hline$<1$ & $7(20.0)$ \\
\hline $1-5$ & $14(40.0)$ \\
\hline $6-10$ & $5(14.3)$ \\
\hline$>10$ & $9(25.7)$ \\
\hline \multicolumn{2}{|l|}{ HIV status $\rrbracket$} \\
\hline Negative & 34 (89.5) \\
\hline Positive & $4(10.5)$ \\
\hline \multicolumn{2}{|l|}{ Monocyte:lymphocyte ratio ${ }^{+}$} \\
\hline Pre-treatment & $0.47(0.33-0.73)$ \\
\hline Post-treatment & $0.25(0.16-0.27)$ \\
\hline \multicolumn{2}{|l|}{ Assessment of CNS involvement } \\
\hline Patients assessed for CNS involvement & 39 (92.9) \\
\hline Imaging of $\mathrm{CNS}^{\S}$ & $28(71.8)$ \\
\hline Head CT performed & $14(50.0)$ \\
\hline CT abnormal & $2(14)$ \\
\hline Head MRI performed & $20(71.4)$ \\
\hline MRI abnormal & $13(65)$ \\
\hline Lumbar puncture performed ${ }^{\S}$ & $31(79.5)$ \\
\hline Lumbar puncture abnormal & $5(16.7)$ \\
\hline \multicolumn{2}{|l|}{ Patients with CNS involvement and test outcome } \\
\hline Total number of patients with CNS involvement ${ }^{\S}$ & 15 (38.5) \\
\hline Lumbar puncture performed in those with CNS involvement & $12(80.0)$ \\
\hline Lumbar puncture sensitivity \% (95\% CI) & $41.7(15.2-72.3)$ \\
\hline Head CT performed in those with CNS involvement $n$ & 5 \\
\hline Head CT sensitivity \% (95\% Cl) & $40.0(5.3-85.3)$ \\
\hline Head MRI performed in those with CNS involvement $\mathrm{n}$ & 14 \\
\hline Head MRI sensitivity \% (95\% CI) & $92.9(66.1-99.8)$ \\
\hline
\end{tabular}

Data are presented as $\mathrm{n}(\%)$ or median (interquartile range), unless otherwise stated. CNS: central nervous system; CT: computed tomography; MRI: magnetic resonance imaging. \#: data available for 35 patients; ๆ: four patients did not have a HIV test; $^{+}$: data available for 23 patients; ${ }^{\S}$ : denominator is 39 patients.

national mortality figures (11.7\% in 2011) [6]. However, most of the large case series have been conducted in the developing world with differing resources, TB prevalence and socioeconomic factors predisposing to the development of miliary TB.

A relatively high proportion of patients in this developed world setting had evidence of CNS involvement on imaging and/or lumbar puncture, which is in keeping with the known pathology and complications of miliary TB [7]. Our findings strongly indicate that, even if an initial head CT is performed for simplicity and logistical reasons, brain MRI should be performed in all patients with miliary TB in resource-rich settings, irrespective of the presence of neurological manifestations. This is in stark contrast to current NICE guidelines, which only recommend neuroimaging for patients with CNS symptoms or signs [5]. The utility of lumbar puncture as a diagnostic tool in previous studies varies greatly and was often restricted to 
those with neurological features; culture positivity rates from CSF ranged from $30 \%$ to $60 \%$ in previous studies in comparison to $13 \%$ (four out of 30 patients) in our study. Patients in this study may have presented earlier, and there was a clear discrepancy between MRI results that showed abnormalities in $65 \%$ of patients compared to lumbar punctures which were only abnormal in $16.7 \%$, suggesting that CSF changes occur relatively late in the presentation of CNS disease in miliary TB.

This study showed that the monocyte:lymphocyte ratio was raised at presentation/pre-treatment and significantly decreased at the end of treatment. SABIN et al. [8] showed that the monocyte:lymphocyte ratio was predictive of the severity of experimental Mycobacterium bovis infection in rabbits. This observation has recently been followed up in large cohorts of HIV co-infected patients in South Africa and active TB patients in China, where extremes in monocyte:lymphocyte ratio predicted subsequent development of TB in this group and the initiation of TB therapy significantly reduced the monocyte:lymphocyte ratio $[9,10]$. These observations highlight the importance of the monocyte:lymphocyte ratio in TB pathogenesis; whether these changes are a product of TB infection itself, or a reflection of individual predisposition to development of active disease requires further evaluation in diverse populations and TB phenotypes.

In conclusion, we emphasise the importance of awareness and early diagnostic investigations in high-risk groups to confirm the diagnosis and identification of CNS involvement with a brain MRI in all patients with miliary TB. This clinical approach is important as it allows the optimal management, particularly in terms of the duration of drug treatment, of patients with neurological involvement thereby preventing relapse and augmenting efforts to achieve TB elimination [11, 12]. Despite a high prevalence of co-existing CNS involvement, overall mortality was low; this may have been due to a high index of suspicion in an ethnically diverse population and early initiation of therapy [13]. Obtaining specimens for culture is imperative and has a high yield. Finally, the monocyte:lymphocyte ratio appears to be of value in monitoring treatment response and warrants further evaluation in larger studies as a marker of clinical response in patients being treated for active disease.

- @ERSpublications

Miliary TB: high level of CNS involvement best investigated by head MRI which suggests re-evaluation of NICE guidance http://ow.ly/X3CDK

Navin Venkatraman ${ }^{1}$, Thomas King ${ }^{1}$, David Bell ${ }^{1}$, Gerrit Woltmann ${ }^{2}$, Martin Wiselka ${ }^{1}$, Ibrahim Abubakar ${ }^{3,4}$ and Manish Pareek ${ }^{1,4,5,6}$

${ }^{1}$ Dept of Infection and HIV Medicine, University Hospitals of Leicester, Leicester, UK. ${ }^{2}$ Dept of Respiratory Medicine, University Hospitals of Leicester, Leicester, UK. ${ }^{3}$ Research Dept of Infection and Population Health, University College London, London, UK. ${ }^{4}$ TB Section, Public Health England, London, UK. ${ }^{5}$ Dept of Infection, Immunity and Inflammation, University of Leicester, Leicester, UK. ${ }^{6}$ Dept of Health Sciences, University of Leicester, Leicester, UK.

Correspondence: Manish Pareek, Dept of Infection, Immunity and Inflammation, University of Leicester, Jarvis Building, Leicester Royal Infirmary, Leicester, LE1 5WW, UK. E-mail: mp426@le.ac.uk

Received: Oct 142015 | Accepted after revision: Dec 202015 | First published online: Feb 042016

Conflict of interest: None declared.

\section{References}

World Health Organization. Global Tuberculosis Report. Geneva, WHO, 2013.

Abdallah FCB, Ali GB, Mahouachi R, et al. Miliary tuberculosis: about 16 cases. Eur Respir J 2011; 38: Suppl 55, P2578.

3 Zaghba N, Bakhatar A, Yassine N, et al. Clinical, epidemiological and evolving profile of miliary tuberculosis: Study of 48 cases. Eur Respir J 2011; 38: Suppl 55, P2572.

4 Pankratova L, Kazimirova N. Modern social and clinical characteristics of miliary tuberculosis. Eur Respir J 2014; 44: Suppl 58, P2630.

5 National Institute for Health and Clinical Excellence. Tuberculosis: clinical diagnosis and management of tuberculosis, and measures for its prevention and control. London, NICE, 2011.

6 Public Health England. Treatment outcome at 12 months by site of disease, UK, 2011. http://webarchive. nationalarchives.gov.uk/20140714084352/http://www.hpa.org.uk/webc/HPAwebFile/HPAweb_C/1317139694968 Date last accessed: February 5, 2014.

7 Sharma SK, Mohan A, Sharma A, et al. Miliary tuberculosis: new insights into an old disease. Lancet Infect Dis 2005; 5: 415-430.

8 Sabin FR, Doan CA, Cunningham RS. Studies of the blood in experimental tuberculosis: the monocytelymphocyte ratio; the anemia-leucopenia phase. Trans 22nd Annu Meet Natl Tuberc Assoc 1926; 22: 252-256.

9 Naranbhai V, Hill AV, Abdool Karim SS, et al. Ratio of monocytes to lymphocytes in peripheral blood identifies adults at risk of incident tuberculosis among HIV-infected adults initiating antiretroviral therapy. J Infect Dis 2014; 209: 500-509.

10 Wang J, Yin Y, Wang X, et al. Ratio of monocytes to lymphocytes in peripheral blood in patients diagnosed with active tuberculosis. Braz J Infect Dis 2015; 19: 125-131.

11 D'Ambrosio L, Dara M, Tadolini M, et al. Tuberculosis elimination: theory and practice in Europe. Eur Respir J 2014; 43: 1410-1420. 
12 Lönnroth K, Migliori GB, Abubaker I, et al. Towards tuberculosis elimination: an action framework for low-incidence countries. Eur Respir J 2015; 45: 928-952.

13 Aouadi S, Mjid M, Maalej S, et al. Miliary tuberculosis: diagnosis difficulties and prognosis factors. Eur Respir J 2011; 38: Suppl 55, P2579.

\section{First experience of effectiveness and safety of bedaquiline for 18 months within an optimised regimen for XDR-TB}

To the Editor:

Extensively drug-resistant (XDR) tuberculosis (TB) is a type of multidrug-resistant (MDR) TB that is resistant to isoniazid, rifampicin, fluoroquinolones and at least one injectable second-line drug. There are insufficient antibiotics for effective combination therapy and mortality exceeds $70 \%$ [1]. Following successful phase IIb trials [2] in 2013, the novel mycobacterial ATP-synthase inhibitor bedaquiline was approved in Europe and the USA for the first 24 weeks of MDR/XDR-TB treatment alongside a World Health Organization (WHO)-approved optimised background regimen. Phase III trials are ongoing but cohort data describe good early bacteriological outcomes in France [3, 4], Italy [5], the UK [6], the USA [7], India [8] and South Africa [9].

However, WHO guidelines presently advocate a total MDR/XDR-TB treatment duration of $\geqslant 18$ months. In the presence of extensive resistance, antibiotic regimens may be sparse when bedaquiline is stopped. The US Centers for Disease Control and Prevention (CDC) recommend continuing bedaquiline beyond 24 weeks if effective treatment "cannot otherwise be provided", whilst acknowledging the lack of supporting tolerability data [10]. Although no fatalities in pre-licensing studies were attributed to the study drug, there were more deaths on bedaquiline than placebo (10 versus two) [2]. The plasma half-life of bedaquiline is $4-5$ months and corrected QT interval (QTc) prolongation occurs, raising concerns about incremental toxicity and highlighting the need for expedient reporting of clinical experience. Here, we describe the first published case of safe bedaquiline treatment extension to 18 months in a patient with pulmonary XDR-TB.

In January 2014, a 20-year-old, HIV-negative Romanian woman was referred to our unit with a first presentation of smear-positive pulmonary TB. Computed tomography (CT) scans showed multifocal disease of both lungs. She had no comorbidities and was initiated on first-line antituberculous chemotherapy (rifampicin, isoniazid, pyrazinamide and ethambutol) but after 2 days, the Genotype MDRTBplus 2.0 assay (Hain Diagnostics, Cookville, TN, USA), performed on the patient's sputum sample, revealed rpoB and kat $G$ mutations consistent with rifampicin and isoniazid resistance. Therefore, she was switched to a WHO-approved MDR-TB regimen (figure 1). During February, full phenotypic drug susceptibility testing and whole-genome sequencing revealed extensive resistance to all first-line drugs, all quinolones, all injectables and prothionamide. Susceptibilities to linezolid, para-aminosalicylic acid (PAS) and azithromycin were confirmed. Phenotypic sensitivity testing to meropenem/imipenem and clofazimine were unavailable, but whole-genome sequencing was performed and identified $k a t G$ mutations conferring high-level isoniazid resistance, precluding the use of high-dose isoniazid. At week 8 of treatment, she was established on a six-drug regimen of bedaquiline, linezolid, pyrazinamide, cycloserine, azithromycin and PAS. Bedaquiline was obtained through a compassionate use programme and introduced at $400 \mathrm{mg}$ once daily for 2 weeks, followed by $200 \mathrm{mg}$ three times weekly. Figure 1 illustrates progression of her regimen as resistance information became available and throughout the remainder of therapy. Her sputum converted to smear and culture negative 24 days after initiation of the bedaquiline based regimen, and 81 days after starting first-line treatment.

She successfully completed her initial supply of bedaquiline. Between week 32 and 34, she developed painful peripheral neuropathy, confirmed on nerve conduction studies, which prompted discontinuation of linezolid and cycloserine. After discussion with the British Thoracic Society MDR-TB advisory panel and international experts, her remaining regimen was deemed inadequate and her bilateral pulmonary disease 